\title{
Paulina Heil
}

\section{Boxine GmbH - Kein Erfolg ist kinderleicht}

Das Unternehmen Boxine GmbH gründeten Patric Faßbender und Marcus Stahl im Dezember 2013 in Düsseldorf. Sie hatten ein innovatives Produkt an der Schnittstelle zwischen Kinderspielzeug und Audiogerät für Kinder kreiert, das sie „Toniebox“ nannten (Abb. 1.1). Dabei handelt es sich um ein elektronisches Gerät in Form eines farbenfrohen, gepolsterten Würfels, das Musik, Hörbücher sowie andere Audiodateien abspielen und auf dem man spezielle Hörspielfiguren („Tonies“) platzieren kann. Um eine Nutzung für Kinder ab einem Alter von drei Jahren zu ermöglichen, musste die Box einfach und sicher zu bedienen sein. Die Boxine $\mathrm{GmbH}$ hatte zum Zeitpunkt der Unternehmensgründung weder in Deutschland noch auf dem internationalen Markt direkte Wettbewerber.

Nachfolgend stellt die Fallstudie die Entwicklung der Boxine GmbH seit ihrer Gründung ausführlich dar in Verbindung zur Entwicklung der relevanten Teilmärkte (Musik, Hörbücher sowie Audioprodukte für Kinder). Schwerpunkt wird die Analyse der Herausforderungen sein, mit denen die Boxine GmbH von Anfang an konfrontiert war, sowie die Lösungsstrategien, die das Team dafür angewendet hat. Zuletzt erfolgt die Zusammenfassung der Analyseergebnisse und ein kurzer Ausblick.

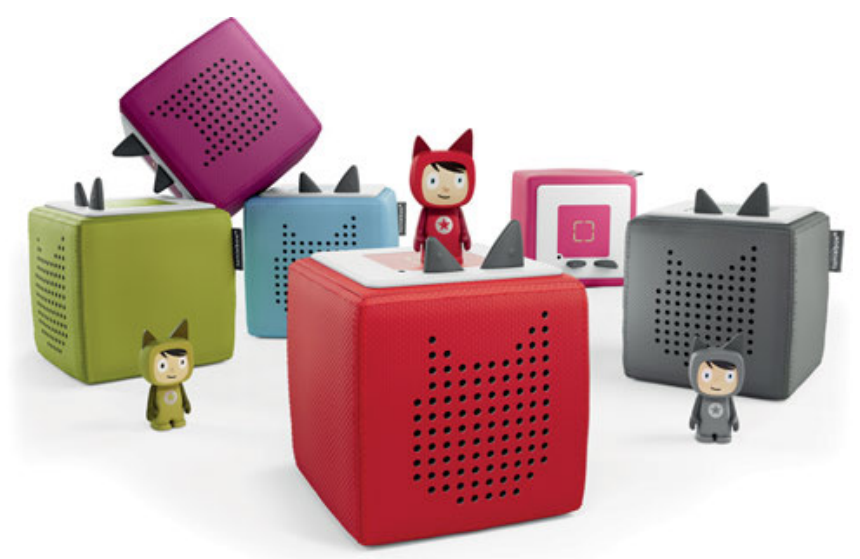

Abb. 1.1: Tonieboxen mit Tonies (Bildnachweis: tonies.de) 


\section{Entwicklung der Teilmärkte für Musik, Hörbücher und Audioprodukte für Kinder}

Die Boxine GmbH hat dank einer innovativen Gründungsidee eine Marktnische geschaffen, die es zuvor nicht gab. Die für das Unternehmen relevante Marktsegmente sind Musik, Hörbücher sowie Audioprodukte für Kinder (Abb. 1.2).



Abb. 1.2: Zuordnung der Produktion der Boxine $\mathrm{GmbH}$ zu den relevanten Marktsegmenten (eigene Darstellung)

Diese Marktsegmente entwickelten sich in Deutschland in den Jahren 2016 und 2017 besonders dynamisch (Abb. 1.3). Der Umsatz von physischen Tonträgern wie zum Beispiel CDs sank von 989 Millionen Euro im Jahr 2016 auf 848 Millionen Euro im Jahr 2017. Im Gegensatz dazu stieg der Umsatz von digitalem, also nicht physischem Musikverkauf wie zum Beispiel Audiostreaming oder Downloadservices in demselben Zeitraum von 604 Millionen Euro auf 741 Millionen Euro an. Die Entwicklung des Umsatzes von speziell für Kinder entwickelte Audioprodukte verlief ebenfalls positiv: Er wuchs von 138 Millionen Euro im Jahr 2016 auf 149 Millionen Euro im Jahr 2017.

Ein negativer Trend ist bei dem Umsatz von Hörbüchern in Deutschland zu beobachten: Er ging von 85 Millionen Euro im Jahr 2016 auf 80 Millionen Euro im Jahr 2017 zurück. Zum Vergleich: In den USA - dem weltweit größten Markt für die digitale Verlagsbranche - nahm der Umsatz von Hörbüchern in derselben Zeitspanne von 2,1 Milliarden US-Dollar auf 2,5 Milliarden US-Dollar zu. ${ }^{1}$

1 Vgl. Statista (2019), verfügbar unter https://www.statista.com/statistics/249854/audiobookindustry-size-in-the-us/. 


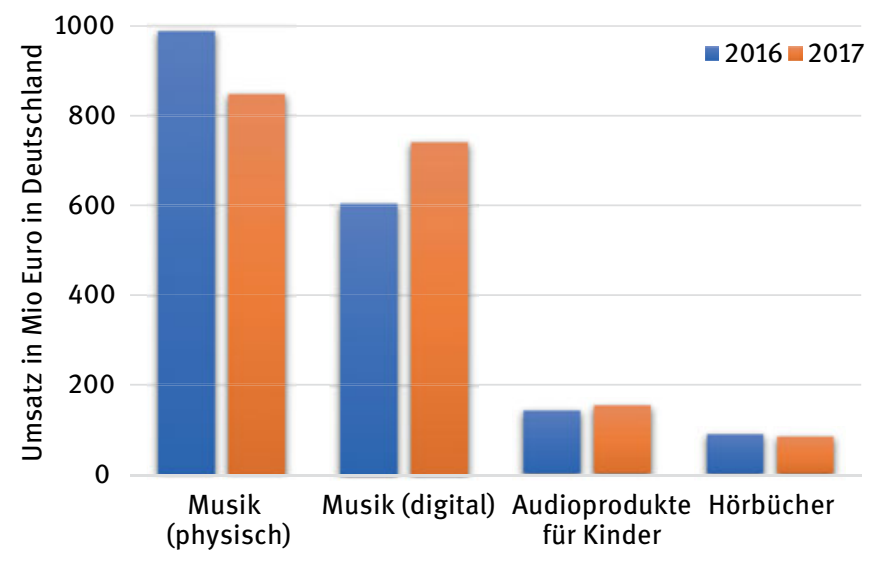

Abb. 1.3: Umsatzentwicklung in Millionen Euro in den Teilmärkten Musik, Audioprodukte für Kinder und Hörbücher in Deutschland von 2016 und 2017 (Quelle: Musikindustie in Zahlen (2017), verfügbar unter http://www.musikindustrie.de/fileadmin/bvmi/upload/02_MarktBestseller/MiZ-Grafiken/2017/BVMI_ePaper_2017.pdf)

Um die Marktsituation in Deutschland besser verstehen zu können, ist es wichtig, sich auch mit der Altersstruktur der Hörbuchkäufer auseinanderzusetzen. Die größte Käufergruppe stellten im Jahr 2016 mit ca. 40 Prozent die 40- bis 59-Jährigen dar. Die Daten lassen keine differenzierte Betrachtung zu, welche Hörbuchkategorie sie am häufigsten kauften. Da diese Altersgruppe von einer Familiensituation dominiert wird, darf man annehmen, dass ein Teil der von ihnen gekauften Hörbücher für Kinder bestimmt war. Bei den 10- bis 19-Jährigen ist der Anteil von den Hörbuchkäufern in den Jahren 2015 und 2016 von ca. 4 Prozent auf 9 Prozent gestiegen. ${ }^{2}$ Dieser Zuwachs ist insbesondere vor dem Hintergrund der vergleichsweise geringen Kaufkraft dieser Altersklasse bedeutend.

Stahl vertritt die Meinung, dass die Boxine GmbH keinem großen Wettbewerbsdruck ausgesetzt ist (Stand: Juni 2018). Das erste und bislang einzige direkte Konkurrenzprodukt präsentierte das Hamburger Unternehmen Tiger Media Deutschland GmbH erst im Jahr 2017 auf der Frankfurter Buchmesse: Die tigerbox ist ein Multifunktionslautsprecher, der ausgesuchte Inhalte auf einer Hörspiel- und Musikplattform (tigertones) abspielt. Optisch ist die tigerbox der Toniebox ähnlich, aber alle tigerboxen bestehen aus Holz und haben eine gummierte, rutschfeste Oberfläche. Die tigerbox warb mit über 1.000 Hörspielen und Musiktiteln, die bereits im Startpaket enthalten waren, sie hat jedoch keine eigene Spiel-

2 Vgl. Analyse des Hörbuchmarkts (2017), verfügbar unter https://www.boersenblatt.net/artikelanalyse_des_gfk_consumer_panels.1298661.html. 
figur, das heißt kein Äquivalent zum Tonie. Die Preise für verschiedene tigerboxEditionen variieren zwischen 26,99 Euro und 34,99 Euro, ${ }^{3}$ während eine Toniebox für 79,95 Euro deutlich teurer war. ${ }^{4}$

\section{Von der Gründungsidee zum Unternehmen}

Die Gründungsidee stammt von Faßbender: Als er seinen Kindern beim Spielen zusah, fragte er sich, wie es sein kann, dass es keine Möglichkeit gab, Audioprodukte für Kinder wie zum Beispiel Hörbücher, Hörspiele und Musik wiederzugeben. Digitale Medien wurden bis dato auf Geräten wie Smartphones, Tablets und Computern gespeichert und wiedergegeben. Diese Situation brachte insbesondere für Kleinkinder eine Reihe von Nachteilen mit sich, zum Beispiel Schwierigkeiten bei der Bedienung, ständige elterliche Kontrolle und Reizüberflutung wegen visueller Eindrücke aufgrund blinkender Bildschirme. Zudem sind physische Tonträger wie zum Beispiel CDs nicht für das Spielen von Kindern geeignet.

Faßbender setzte sich zusammen mit seinem Freund Stahl, der ebenfalls Vater ist, das Ziel, diese Situation zu ändern. Sie entwickelten einen Tonie, also eine Hörfigur, die man auf einen gepolsterten Würfel mit der notwendigen elektronischen Ausstattung, der Toniebox, stellen kann, um Hörspiele, Musik und andere Audiodateien abzuspielen. Sie sollte für Kinder ab einem Alter von drei Jahren einfach zu bedienen, dabei robust und sicher sein sowie den natürlichen Spieltrieb unterstützen. Der Tonie ist bei Hörspielen der Protagonist der jeweiligen Geschichte, die das Kind hört. Zusätzlich gibt es Tonies, die Liedersammlungen beinhalten, und Kreativ-Tonies, die von den Kunden/innen selbst bespielt werden können.

Um diese Idee umzusetzen, gründeten Faßbender und Stahl im Dezember 2013 das Unternehmen Boxine GmbH. Zuvor war Faßbender jahrelang als Kreativdirektor einer großen Agentur tätig gewesen, während Stahl in einer Automobilzulieferergesellschaft gearbeitet hatte. Vor der Gründung der Boxine GmbH hatte Stahl ein Geschäft im Sinne vom Buy-out übernommen. Eine Gründungserfahrung im engeren Sinne konnten beide nicht vorweisen. Ebenfalls hatte keiner von ihnen in der Vergangenheit bei einem Unternehmen gearbeitet, das Kinderprodukte vermarktete.

3 Vgl. Hörspielspaß im Kinderzimmer mit der tigerbox (2017), verfügbar unter https://tiger. media/wp-content/uploads/2017/10/PM_tigertones_tigerbox_n.pdf.

4 Vgl. Boxine GmbH (2018), verfügbar unter https://tonies.de/. 
Obwohl die Gründer sowohl mit dem Start-up als auch mit der Branche ein für sie komplett neues Terrain betraten, erlebten sie die Gründung von Boxine $\mathrm{GmbH}$ nicht als einen „Sprung ins kalte Wasser“. Stahl erklärt: „Wenn die Entscheidung getroffen ist, dann ist das alternativlos und man arbeitet täglich mit einer großen Leidenschaft und Energie daran. Ich hatte nie eine schönere Zeit. “ Neben der Leidenschaft für das eigene innovative Produkt habe laut Stahl die Unterstützung der Familie, die die beiden Gründer von Anfang an gehabt haben, eine wichtige Rolle gespielt.

Faßbender und Stahl sehen sich als „Düsseldorfer“ und lieben die Stadt am Rhein. Sie leben hier seit vielen Jahren mit ihren Familien und verfügen über ein breites soziales Netzwerk vor Ort, das ihnen unter anderem für die Gründung ihres Unternehmens hilfreich war. Die Stadt bietet vielfältige Start-up-Events an. Hier stellen potenzielle Gründer ihre Ideen vor einer aus Investoren bestehenden Jury vor, tauschen Erfahrungen aus und knüpfen nützliche Kontakte. Allerdings sieht Stahl an dieser Stelle noch viel Verbesserungspotenzial: „Bei wirklich wichtigen Dingen, die so eine Gründung braucht, nämlich Geld und Räumlichkeiten, müsste Düsseldorf viel nachholen. “ Zusätzlich würden die potenziellen Gründer seiner Erfahrung nach von einer Beratung in Rechtsfragen profitieren, die die Stadt anbieten stellen könnte.

An anderen beliebten Start-up-Standorten in Deutschland wie zum Beispiel in Berlin habe, so Stahl, zum Gründungszeitpunkt eine ähnliche Situation geherrscht. Er erklärt das damit, dass in Deutschland eine risikovermeidende Mentalität besteht: Banken sowie die Städte seien hierzulande nicht bereit, in innovative und somit risikobehaftete Gründungen $\mathrm{zu}$ investieren. Bei weniger innovativen und deshalb sichereren Unternehmensgründungen, zum Beispiel die Eröffnung eines Eiscafés, sei die Investitionsbereitschaft seitens der Stadt und der Banken seiner Meinung nach erheblich höher. Auch die Gründer der Boxine $\mathrm{GmbH}$ hatten zu Beginn Schwierigkeiten damit, potenzielle Investoren von ihrer Idee zu überzeugen.

Vor dem Hintergrund der skizzierten Investitionssituation in Deutschland entschieden sich Faßbender und Stahl dafür, die Gründung der Boxine GmbH mit Eigenkapital zu finanzieren. Da die Toniebox ein komplexes Elektronikprodukt darstellt und somit einen anspruchsvolleren Herstellungsprozess als zum Beispiel eine App erfordert, waren die Kosten für die Produktentwicklung entsprechend hoch. Von der Unternehmensgründung im Jahr 2013 bis zum Produktlaunch im Jahr 2016 trugen die Gründer 100 Prozent der Kosten. Später schlossen sie einen Gesellschaftsvertrag ab und übergaben Anteile an die neuen Gesellschaftsmitglieder, die sie über ihr soziales Netzwerk akquirieren konnten. Zusätzlich bemühten sich beide um die Finanzierung durch diverse Banken in Düsseldorf. 
Die Boxine GmbH entwickelte sich rasant von einem Start-up, dessen Team im Jahr 2013 lediglich aus den beiden Gründern bestand, zu einem erfolgreichen jungen Unternehmen, für das im Jahr 2018 am Standort Düsseldorf 80 Mitarbeiter/ innen tätig sind. Die ersten fanden die Gründer im eigenen sozialen Netzwerk, das auch später die wichtigste Ressource für die Mitarbeiterrekrutierung blieb. Weiteres Personal fanden sie über Initiativbewerbungen, spezialisierte Headhunter für IT-Fachkräfte und Stellenanzeigen. Entgegen der verbreiteten Meinung, junge Unternehmen seien insbesondere für Hochschulabsolventen attraktiv, besteht das Team der Boxine $\mathrm{GmbH}$ aus Mitarbeitern/innen verschiedenen Alters zwischen 20 und 60 Jahren (Stand: Juni 2018). Stahl zufolge liegt dem Unternehmen „eine gute Mischung aus vielen erfahrenen Leuten und vielen jungen Leuten“ zugrunde.

Der Umsatz entwickelte sich positiv. Nach dem Produktlaunch und dem ersten Weihnachtsgeschäft im Jahr 2016 umfasste der Umsatz über 2 Millionen Euro. Im ersten vollen Geschäftsjahr 2017 erzielte die Boxine GmbH rund 17 Millionen Euro Umsatz. Im Jahr 2018 lag der Umsatz bei 60 Millionen Euro (Abb. 1.4).

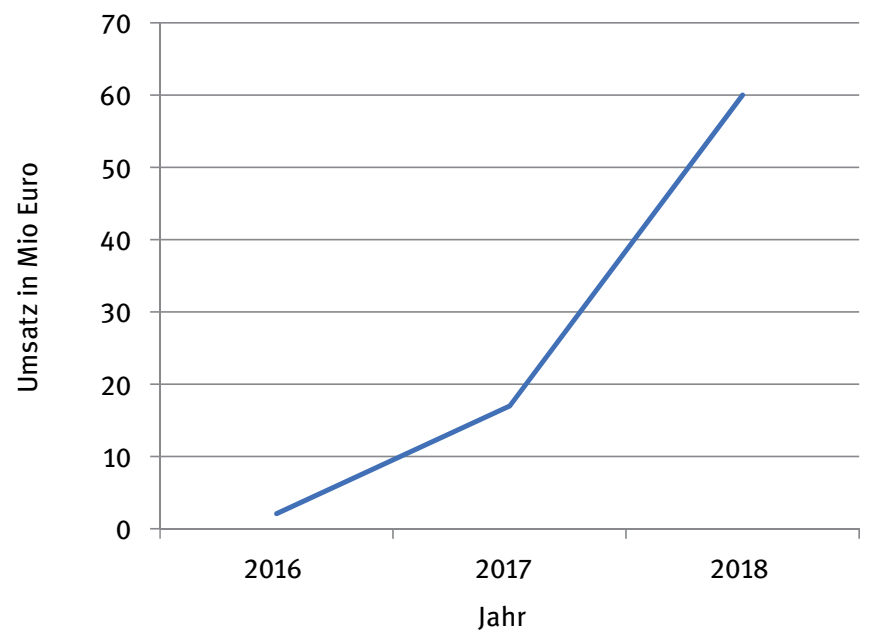

Abb. 1.4: Umsatzentwicklung der Boxine GmbH in Millionen Euro zwischen 2016 und 2018 (eigene Darstellung)

Die Expansion auf internationale Märkte ist ein wichtiges strategisches Ziel der Boxine GmbH. Stahl dazu: „Wir wollen wirklich, dass die Tonies in jedes Kinderzimmer weltweit kommen.“ Die Situation scheint dafür günstig zu sein, da der weltweit größte Hörbuchmarkt in den USA sich in den letzten Jahren positiv ent- 
wickelte und die Boxine $\mathrm{GmbH}$ national wie international kaum direkte Konkurrenten hat. Vor diesem Hintergrund wagte das Unternehmen im Jahr 2018 einen ersten Schritt über Deutschlands Grenzen hinaus und eröffnete eine Zweigniederlassung in London. In Zukunft sollen die Märkte in den USA, Asien und europäischen Ländern erschlossen werden.

\section{Gute Idee, gutes Konzept}

Das Konzept für die Toniebox stand bereits bei der Unternehmensgründung fest. Aufgrund der konkreten Gestaltung der Toniebox ergaben sich jedoch einige Fragen, für die es aufgrund der Innovation des Produkts noch keine fertigen Lösungen gab. Eine besonders wichtige Rolle spielte dabei die Oberflächengestaltung. Um das Produkt für Kinder ansprechend zu gestalten, sollte die Oberfläche der Toniebox weich gepolstert sein. Doch diese Idee brachte zahlreiche Anforderungen mit sich:

- Sicherheit: Sowohl die oberste Stoffschicht als auch der Polsterstoff sollten den strengsten Qualitätsrichtlinien entsprechen, um die Gesundheit der Kinder nicht zu gefährden.

- Stabilität: Der Stoff durfte trotz der hohen Belastung beim Spielen (häufiges Anfassen, Ziehen, Fallenlassen etc.) nicht leicht kaputtgehen.

- Optik und Haptik: Der Stoff sollte sich im Hinblick auf die Optik und Haptik ansprechend gestaltet sein, um ein spielerisches Erlebnis bei der Benutzung zu gewährleisten. Zum Beispiel sollte der Stoff in verschiedenen Farben in der gleichen Qualität zur Verfügung stehen.

Einen Stoff zu finden, der alle genannten Kriterien erfüllte, war nicht einfach. Eine Zeit lang diskutierte das Team darüber, stattdessen einen Kunststoff zu verwenden. Die beiden Gründer trafen jedoch die Entscheidung, weiter nach dem geeigneten Stoff und der Polsterung zu suchen, denn er unterstrich die Einzigartigkeit des Produkts. Die Experimente mit der Oberflächengestaltung von der Toniebox nahmen insgesamt ca. elf Monate in Anspruch. Schließlich wurde ein Polsterstoff gefunden, der allen Anforderungen entsprach.

Der Prototyp der Toniebox wurde im Januar 2016 auf einer Spielwarenmesse in Nürnberg präsentiert und beworben. Der Produktlaunch war für Juni 2016 geplant. Als das Team der Boxine GmbH im März 2016 feststellte, dass die technische Ausstattung der Toniebox nicht zuverlässig genug funktionierte, lagen bereits die ersten Kundenbestellungen vor. Nun gab es zwei Optionen:

1. die Aufträge erfüllen und dabei die technische Unfertigkeit in Kauf nehmen oder 
2. die Aufträge vorübergehend auf Eis legen und erst die technische Ausstattung der Toniebox optimieren, sodass sie den höchsten Anforderungen entsprach.

Die erste Option hatte einen kurzfristigen finanziellen Vorteil, da die Kunden/ innen die erworbenen Produkte bezahlen würden und so ein Umsatz erfolgte. Gleichzeitig brachte sie das Risiko mit sich, die Kunden/innen aufgrund einer unzureichenden Produktqualität zu enttäuschen und somit die Unternehmungsreputation dauerhaft zu schädigen.

Bei der zweiten Option verhielt es sich umgekehrt: Kurzfristig bestand die Gefahr, finanzielle Ressourcen zu verlieren, da die Kunden/innen kein Produkt bezahlen würden, das ihnen nicht rechtzeitig zur Verfügung stünde. Die Ausgaben für die fortwährende Produktmodifizierung und die Mitarbeitergehälter blieben währenddessen bestehen. Langfristig sollte jedoch die reibungslos funktionierende technische Ausstattung der Toniebox die Kundenzufriedenheit etablieren, steigern und einen Grundstein für die hervorragende Unternehmensreputation legen. Diese sollte wiederrum den langfristigen Zugang zu finanziellen Ressourcen und die weitere Entwicklung des Unternehmens sichern.

\section{Zum Vergleich mit Global Playern}

In solchen Entscheidungssituationen befinden sich keinesfalls allein junge Unternehmen. Vielmehr sind alle Unternehmen (unabhängig von Alter und Größe) damit konfrontiert, sich mit der Entwicklung von innovativen Produkten auseinanderzusetzen. Es bietet sich ein international etabliertes Unternehmen für einen Vergleich an, das ebenfalls auf die Entwicklung technischer Innovationen angewiesen ist, nämlich Microsoft.

Der Softwarekonzern beschäftigte sich ab dem Jahr 2001 mit der Entwicklung der Version Windows Vista und brachte das Produkt am 30. Januar 2007 auf den Markt. ${ }^{5}$ Nach einigen Schätzungen investierte das Unternehmen 500 Millionen US-Dollar in die Marketingkampagne und rechnete damit, dass die Hälfte der Anwender innerhalb der darauf folgenden zwei Jahre die Premiumversion nutzen würde. ${ }^{6}$

Trotz der relativ langen Entwicklungszeit, der relativ hohen Entwicklungskosten und einer aggressiven Marketingkampagne entsprach Windows Vista

5 Vgl. Facts About Microsoft (2018), verfügbar unter https://news.microsoft.com/facts-aboutmicrosoft/.

6 Vgl. AdAge (2007), verfügbar unter http://adage.com/article/digital/microsoft-pumps-500million-vista-marketing-campaign/114589/. 
nicht den Anforderungen der Kunden/innen, denn sie nahmen es als ein Produkt wahr, das für eine Vermarktung noch nicht bereit war. Dafür gab es mehrere Gründe. Zum einen hatte sich das Unternehmen das Ziel gesetzt, die Nutzerkonten so sicher wie möglich zu machen. Infolgedessen wurden die Nutzer selbst bei trivialen Operationen wie dem Öffnen eines Programms oder einer Datei mit Systemmeldungen konfrontiert und mussten das Fortführen der jeweiligen Operation zunächst bestätigen. Daraus ergab sich insgesamt eine zu hohe Anzahl von Systemmeldungen, die den Arbeitsfluss störten.

Außerdem beanspruchte Windows Vista eine zu große Speicherkapazität vieler Computerfestplatten, die zu diesem Zeitpunkt auf dem Markt waren, das heißt, bei älteren Geräten kam es zu Kompatibilitätsproblemen zwischen der neuen Software und der bestehenden Hardware. Aufgrund der fehlenden technischen Kompatibilität erlebten viele Nutzer die Arbeit mit dem neuen System als zu langsam und instabil.

Dabei war Windows Vista nicht günstig. So kostete die Version Windows Vista Ultimate ca. 400 US-Dollar und Vista Home Premium 239 US-Dollar (zum Vergleich: Windows 10 Pro, eine spätere Version mit vergleichbaren Features, kostete nur 199 US-Dollar). Die hohen Kosten und die sich schnell verbreitende negative Kritik führten zu der Entscheidung vieler Nutzer, ihr System nicht gegen Windows Vista zu tauschen. Die Einnahmen des Softwarekonzerns konnten somit nicht das anvisierte Niveau erreichen. ${ }^{7}$

Der gescheiterte Produktlaunch von Windows Vista wurde von dem direkten Konkurrenten Apple in seiner Werbekampagne in der Serie „I'm a Mac“ ausgenutzt. Nach einigen Einschätzungen wäre der Schaden für Microsoft noch größer gewesen, wenn zu dem Zeitpunkt soziale Netzwerke bereits etabliert gewesen wären, da sie zur Verbreitung der negativen Nutzerkritik erheblich beigetragen hätten. ${ }^{8}$ Diese These stützen Nutzerstatistiken: Im Dezember 2006, kurz vor dem Launch von Windows Vista, hatte der Vorreiter auf dem Gebiet der sozialen Medien Facebook weltweit 12 Millionen aktive Nutzer monatlich, während ihre Anzahl im Juni 2018 bereits 2,23 Milliarden betrug. ${ }^{9}$

7 Vgl. Windows Central (2018), verfügbar unter https://www.windowscentral.com/windowsvista-5-things-you-might-not-know-about-microsoft-messiest-os-release.

8 Vgl. Spiegelonline (2011), verfügbar unter http://www.spiegel.de/karriere/a-786369.html.

9 Vgl. Newsroom Facebook - Company Info (2018), verfügbar unter https://newsroom.fb.com/ company-info/. 


\section{Vergangenheit, Gegenwart, Zukunft}

Die Boxine GmbH wurde im Dezember 2013 von Faßbender und Stahl in Düsseldorf gegründet. Das junge Unternehmen wollte ein innovatives Produkt, die Toniebox, entwickeln und somit eine neue, bisher nicht existierende Nische auf dem globalen Markt besetzen. Seit seiner Gründung musste das Unternehmen viele Herausforderungen überwinden, um zum Beispiel eine staatliche Förderung oder einen Bankenkredit als Startfinanzierung zu erhalten. Der Grund dafür habe, so Stahl, in der durch die deutsche Mentalität bedingte Neigung der potenziellen Investoren bestanden, Risiko zu vermeiden. Deshalb mussten er und Faßbender die Unternehmensgründung mit Eigenkapital finanzieren. Auch bei der Suche nach geeigneten kostengünstigen Büroräumen und bei rechtlichen Fragen hätten sie sich mehr Unterstützung seitens der Stadt Düsseldorf gewünscht, als sie zum Zeitpunkt der Gründung erhalten haben.

Weitere Hürden ergaben sich im Zuge der Produktentwicklung: Die Toniebox musste für Kinder in einem Alter ab drei Jahren einfach und sicher zu benutzen sein, technisch reibungslos funktionieren und gleichzeitig als Spielzeug optisch attraktiv gestaltet sein. Diese Schwierigkeiten haben die Gründer gemeistert, indem sie sich für die hohe Produktqualität und nicht für den kurzfristigen Profit entschieden. Die positiven Folgen dieser Entscheidung führten das Unternehmen zum wirtschaftlichen Erfolg: Umsatz und Gewinn stiegen rasant an, während die Anzahl technischer Reklamationen gering blieb. Dieser Erfolg innerhalb Deutschlands erlaubt der Boxine $\mathrm{GmbH}$, für die Zukunft eine internationale Expansionskampagne zu planen. Einen ersten Schritt in diese Richtung ging das Unternehmen, indem es 2018 eine Zweigniederlassung in London eröffnete.

Auch die Zahl der Mitarbeiter/innen entwickelten sich positiv: Aus dem Duo der Gründer im Jahr 2013 ist ein Team aus ca. 80 Personen im Jahr 2018 geworden. Im Unternehmen wird Wert darauf gelegt, eine partizipative Kultur aufrechtzuerhalten. Diese steigert sowohl seine Attraktivität als Arbeitgeber als auch die Motivation der Mitarbeiter/innen.

Die steigenden Verkaufszahlen für die digitalen, nicht physischen Tonträger in Deutschland sowie für die Hörbücher auf dem weltweit größten Markt in den USA bilden die Grundlage für eine positive Prognose. Eine direkte Konkurrenz war zum Zeitpunkt der Fallstudienvorbereitung gering (Stand: November 2018). Es ist anzunehmen, dass die Boxine $\mathrm{GmbH}$ aufgrund der bestehenden Nachfrage und des mangelnden Angebots erfolgreich expandieren und auch auf dem internationalen Markt einen guten Umsatz erzielen wird. Stahl rechnet damit, dass wenn das Konzept von Toniebox internationale Bekanntheit erlangt, andere Unternehmen es nachahmen und modifizieren werden. In der langfristigen Per- 
spektive ist es infolgedessen möglich, dass das Angebot in diesem Marktsegment zunehmend vielfältiger, günstiger und der Markt gesättigt sein wird. Dies wird den Wettbewerbsdruck auf die Boxine $\mathrm{GmbH}$ erhöhen, worauf das Unternehmen mit der Erweiterung des Portfolios auf weitere Altersgruppen, zum Beispiel Grundschulkinder, und der Beibehaltung von hohen Qualitätsstandards reagieren könnte. 


\section{COMPEON}

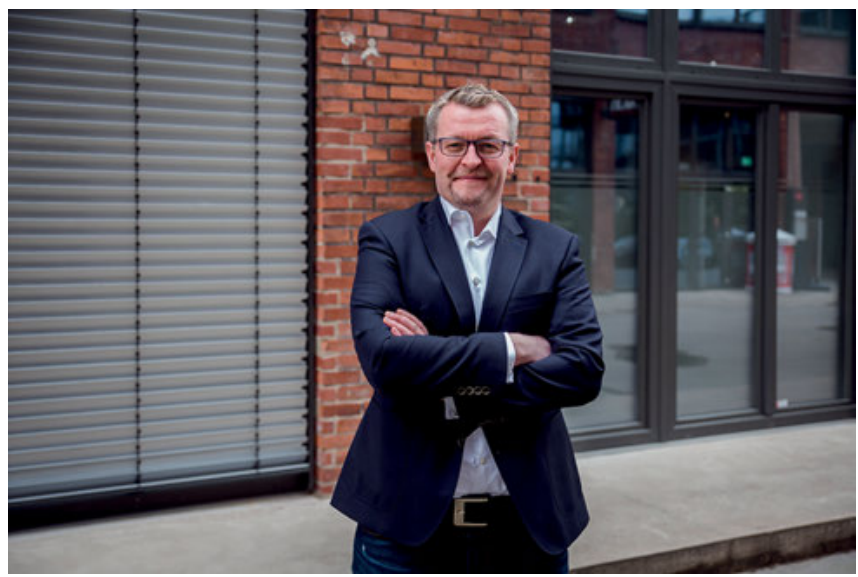

Dr. Frank Wüller - Geschäftsführer der Compeon GmbH (Bildnachweis: Compeon $\mathrm{GmbH}$ )

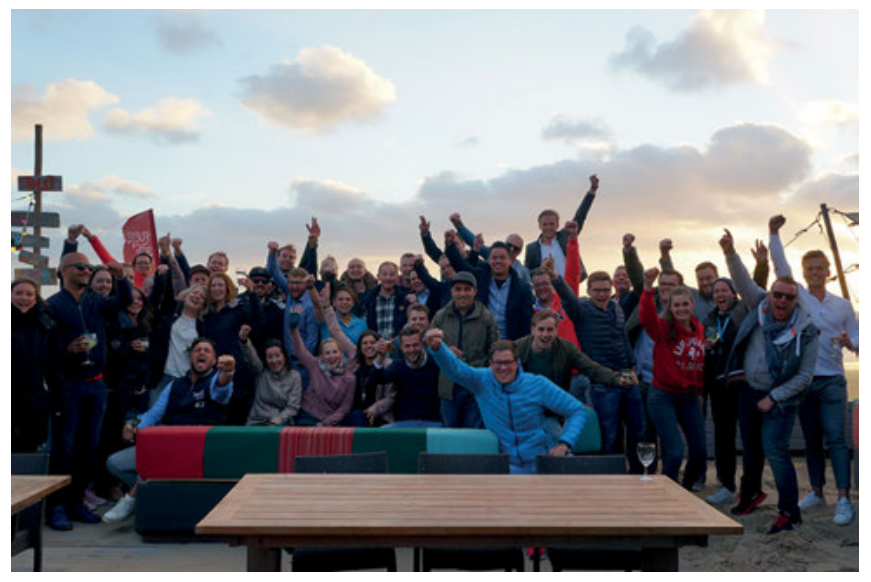

Das Team der Compeon GmbH (Bildnachweis: Compeon GmbH) 